\title{
CERTAIN CONGRUENCE AND QUOTIENT LATTICES RELATED TO COMPLETELY 0-SIMPLE AND PRIMITIVE REGULAR SEMIGROUPS
}

\author{
by H. E. SCHEIBLICH
}

(Received 3 August, 1967; revised 21 May, 1968)

G. Lallement [4] has shown that the lattice of congruences, $\Lambda(S)$, on a completely 0 simple semigroup $S$ is semimodular, thus improving G. B. Preston's result [5] that such a lattice satisfies the Jordan-Dedekind chain condition. More recently, J. M. Howie [2] has given a new and more simple proof of Lallement's result using work due to Tamura [9]. The purpose of this note is to extend the semimodularity result to primitive regular semigroups, to establish a theorem relating certain congruence and quotient lattices, and to provide a theorem for congruences on any regular semigroup.

1. Preliminaries. If $a$ and $b$ are elements of a lattice $L$, then $a$ is said to cover $b(a>b)$ provided that $a>b$ and $a \geqq c \geqq b$ implies that $a=c$ or $b=c$. The lattice $L$ is called semimodular if, whenever $x, y \succ x \wedge y$, then $x \vee y \succ x, y$.

According to Rees [7], every completely 0-simple semigroup is isomorphic to what Clifford and Preston [1] call a Rees $I \times \Lambda$ matrix semigroup $\mathscr{M}^{0}[G, I, \Lambda, P]$ over a groupwith-zero $G^{0}$ with regular sandwich matrix $P$. The condition of regularity on the $\Lambda \times I$ matrix $P=\left(p_{\lambda i}\right)$ over $G^{0}$ is that each row and each column of $P$ contains some nonzero entry.

If $S=\mathscr{M}^{0}[G, I, \Lambda, P]$ is a completely 0 -simple semigroup, then the relation

$$
\varepsilon_{I}=\left\{(i, j) \in I \times I: p_{\lambda i}=0 \text { if and only if } p_{\lambda j}=0\right\}
$$

is an equivalence relation on $I$, as is $\varepsilon_{\Lambda}$, which is defined on $\Lambda$ in an analogous way. If $e$ is the identity of $G$, the matrix $P$ is called normal provided that $i \in J$ implies that there exists $\lambda \in \Lambda$ such that $p_{\lambda l}=p_{\lambda j}=e$ for each $j \in I$ such that $(i, j) \in \varepsilon_{I}$, and similarly $\lambda \in \Lambda$ implies that there exists $i \in I$ such that $p_{\lambda i}=p_{\mu i}=e$ for each $\mu \in \Lambda$ such that $(\lambda, \mu) \in \varepsilon_{\Lambda}$. Tamura [9] has shown that any completely 0 -simple semigroup is isomorphic to a Rees matrix semigroup $\mathscr{M}^{0}[G, I, \Lambda, P]$ in which $P$ is normal. Henceforth, it is assumed that $P$ is normal.

A triple $\left(N, \rho_{I}, \rho_{\Lambda}\right)$ in which $N$ is a normal subgroup of $G$ and $\rho_{I}, \rho_{\Lambda}$ are equivalence relations on $I$ and $\Lambda$, respectively, such that $\rho_{I} \subseteq \varepsilon_{I}$ and $\rho_{\Lambda} \subseteq \varepsilon_{\Lambda}$, is called linked provided that

(i) $(i, j) \in \rho_{I}$ and $p_{\lambda i} \neq 0$ imply $p_{\lambda i} p_{\lambda j}^{-1} \in N$,

(ii) $(\lambda, \mu) \in \rho_{\Lambda}$ and $p_{\lambda i} \neq 0$ imply $p_{\lambda i} p_{\mu i}^{-1} \in N$.

Tamura [9] has shown that there is a map $\Psi$ from the set of linked triples into the set of nontrivial (not universal) congruences on $S$, defined by $((a, i, \lambda),(b, j, \mu)) \in \Psi\left(N, \rho_{I}, \rho_{\Lambda}\right)$ if and only if $a b^{-1} \in N,(i, j) \in \rho_{I},(\lambda, \mu) \in \rho_{\Lambda}$. Moreover, there is a map $\Omega$ from the set of nontrivial congruences on $S$ into the set of linked triples, defined by $\Omega(\rho)=\left(N, \rho_{I}, \rho_{\Lambda}\right)$, where

(i) $a b^{-1} \in N$ if and only if there exist $i, j \in I$ and $\lambda, \mu \in \Lambda$ such that $((a, i, \lambda),(b, j, \mu)) \in \rho$,

(ii) $(i, j) \in \rho_{I}$ if and only if there exist $a, b \in G$ and $\lambda, \mu \in \Lambda$ such that $((a, i, \lambda),(b, j, \mu)) \in \rho$, 
(iii) $(\lambda, \mu) \in \rho_{\Lambda}$ if and only if there exist $a, b \in G$ and $i, j \in I$ such that $((a, i, \lambda),(b, j, \mu)) \in \rho$.

Since $\Psi$ and $\Omega$ are mutually inverse, $\Psi$ is a bijection of the set of linked triples onto the set of nontrivial congruences. The congruence $\Psi\left(N, \rho_{I}, \rho_{\Lambda}\right)$ is denoted by $\left[N, \rho_{I}, \rho_{\Lambda}\right]$.

Howie [2] has shown in his note that, if $\rho=\left[N, \rho_{I}, \rho_{\Lambda}\right]$ and $\sigma=\left[M, \sigma_{I}, \sigma_{\Lambda}\right]$ are congruences on $S$, then

$$
\rho \subseteq \sigma \text { if and only if } M \subseteq N, \rho_{I} \subseteq \sigma_{I}, \rho_{\Lambda} \subseteq \sigma_{\Lambda} .
$$

If $S$ is any regular semigroup, the relation $\theta=\{(\rho, \sigma) \in \Lambda(S) \times \Lambda(S): \rho|E=\sigma| E\}$, where $E$ is the set of idempotents of $S$, is such that each $\theta$ class is a complete modular sublattice of $\Lambda(S)[8]$.

2. A congruence for congruences on a regular semigroup. If $S$ is an inverse semigroup, then the relation $\theta$ defined above on $\Lambda(S)$ is a complete congruence on $\Lambda(S)$ in the sense that $\theta$ is a congruence, $\Lambda(S) / \theta$ is a complete lattice, and $\theta^{\natural}: \Lambda(S) \rightarrow \Lambda(S) / \theta$ is a complete lattice homomorphism [8]. This section will show that this result is true for regular semigroups.

LEMMA 2.1. Let $S$ be a regular semigroup and $\sigma, \tau \in \Lambda(S)$ such that $\sigma$ separates idempotents. Then $(\sigma \vee \tau, \tau) \in \theta$.

Proof. First consider the relation $h=\{(a, b) \in S \times S:(a \tau, b \tau) \in \mathscr{H}\}$. Then $h$ is an equivalence relation on $S$ and a routine check will reveal that $\mathscr{H}, \tau \subseteq h$. To see that $h|E \subseteq \tau| E$, let $(e, f) \in h \mid E$. Then $(e \tau, f \tau) \in \mathscr{H}$ and so $e \tau=f \tau$ since $\mathscr{H}$ separates idempotents in $S / \tau$. Hence $(e, f) \in \tau \mid E$ and so $\tau|E=h| E$.

Now $\sigma$ separates idempotents and so $\sigma \subseteq \mathscr{H}[3$, Theorem 2.3]. Consequently

$$
\tau \subseteq \sigma \vee \tau \subseteq \mathscr{H} \vee \tau \subseteq h \quad \text { and so } \quad \tau|E \subseteq(\sigma \vee \tau)| E \subseteq h|E=\tau| E \text {, }
$$

which implies that $(\sigma \vee \tau, \tau) \in \theta$.

THEOREM 2.2. If $S$ is a regular semigroup, the relation $\theta$ is a complete congruence on $\Lambda(S)$.

Proof. To show that $\theta$ is a complete congruence on $\Lambda(S)$, it is sufficient to show that, if $\left(\rho_{i}, \sigma_{i}\right) \in \theta$ for each $i \in J$, an index set, then both $\left(V \rho_{i}, V \sigma_{i}\right)_{i \in J}$ and $\left(\bigcap p_{i}, \bigcap \sigma_{i}\right)_{i \in J}$ belong to $\theta$. The latter is established by [8, Theorem 5.1].

First, to see that $\theta$ is a congruence on $\Lambda(S)$, suppose that $(\rho, \sigma) \in \theta$ and $\tau \in \Lambda(S)$. Let $\lambda=\bigcap\{\eta \in \Lambda(S): \eta \in \rho \theta=\sigma \theta\}$. Then $\rho / \lambda, \sigma / \lambda$ and $(\tau \vee \lambda) / \lambda$ all belong to $\Lambda(S / \lambda)$ and, furthermore, $\rho / \lambda$ and $\sigma / \lambda$ separate idempotents in the regular semigroup $S / \lambda$. Hence

$$
(\rho / \lambda \vee(\tau \vee \lambda) / \lambda, \sigma / \lambda \vee(\tau \vee \lambda) / \lambda)=((\rho \vee \tau) / \lambda,(\sigma \vee \tau) / \lambda) \in \theta_{s / \lambda}
$$

by Lemma 2.1. Hence $(\rho \vee \tau, \sigma \vee \tau) \in \theta$.

Finally, to see that $\theta$ is complete, assume that $\left(\rho_{i}, \sigma_{i}\right) \in \theta$ for each $i \in J$ and $(e, f) \in\left(\bigvee_{i \in J} p_{i}\right) \mid E$. Then there exist $x_{1}, x_{2}, \ldots, x_{n} \in S$ and $i_{1}, \ldots, i_{n+1} \in J$ such that $\left(e, x_{1}\right) \in \rho_{i_{1}},\left(x_{1}, x_{2}\right) \in \rho_{i_{2}}, \ldots$, $\left(x_{n}, f\right) \in \rho_{i_{n+1}}$. Then 
Symmetrically,

$$
(e, f) \in\left(\bigvee_{j=1}^{n+1} p_{i_{j}}\right)\left|E=\left(\bigvee_{j=1}^{n+1} \sigma_{i_{j}}\right)\right| E \subseteq\left(\bigvee_{i \in J} \sigma_{i}\right) \mid E
$$

$$
\left(\bigvee_{i \in J} \sigma_{i}\right)\left|E \subseteq\left(\bigvee_{i \in J} \rho_{i}\right)\right| E \text { and so }\left(\bigvee \rho_{i}, \bigvee \sigma_{i}\right)_{i \in J} \in \theta
$$

\section{Completely 0-simple and primitive regular semigroups.}

Lemma 3.1. Suppose that $\rho=\left[N, \rho_{I}, \rho_{\Lambda}\right]$ and $\sigma=\left[M, \sigma_{I}, \sigma_{\Lambda}\right]$ are nontrivial congruences on a completely 0-simple semigroup $S=\mathscr{M}^{0}[G, I, \Lambda, P]$. Then $(\rho, \sigma) \in \theta$ if and only if $\rho_{I}=\sigma_{I}$ and $\rho_{\Lambda}=\sigma_{\Lambda}$.

Proof. Assume that $(\rho, \sigma) \in \theta$ and that $(i, j) \in \rho_{I}$. Then there exists $\lambda \in \Lambda$ such that $p_{\lambda i} \neq 0$ and hence, since $\left(N, \rho_{I}, \rho_{\Lambda}\right)$ is a linked triple, $\left(\left(p_{\lambda i}^{-1}, i, \lambda\right),\left(p_{\lambda j}^{-1}, j, \lambda\right)\right) \in \rho|E=\sigma| E$ and so $(i, j) \in \sigma_{I}$. Thus $\rho_{I} \subseteq \sigma_{I}$ and similarly $\sigma_{I} \subseteq \rho_{I}$. Thus $\rho_{I}=\sigma_{I}$ and similarly $\rho_{\Lambda}=\sigma_{\Lambda}$.

Conversely, if $\rho_{I}=\sigma_{I}$ and $\rho_{\Lambda}=\sigma_{\Lambda}$, suppose that $\left(\left(p_{\lambda i}^{-1}, i, \lambda\right),\left(p_{\mu j}^{-1}, j, \mu\right)\right) \in \rho \mid E$. Then $(i, j) \in \rho_{I}=\sigma_{I}$ and $(\lambda, \mu) \in \rho_{\Lambda}=\sigma_{\Lambda}$. Hence $p_{\lambda i} p_{\lambda j}^{-1} \in M$ and $p_{\lambda j} p_{\mu j}^{-1} \in M$, since $\left(M, \sigma_{t}, \sigma_{\Lambda}\right)$ is linked. Thus $p_{\lambda i} p_{\mu j}^{-1} \in M$ and so $\left(\left(p_{\lambda i}^{-1}, i, \lambda\right),\left(p_{\mu j}^{-1}, j, \mu\right)\right) \in \sigma \mid E$. Thus $\rho|E \subseteq \sigma| E$. Similarly, $\sigma|E \subseteq \rho| E$ and so $(\rho, \sigma) \in \theta$.

If $S$ is any regular semigroup, $S$ is called $\theta$-reduced provided that each $\theta$ class is a singleton. A congruence $\rho \in \Lambda(S)$ is called a $\theta$-reduced congruence provided that $S / \rho$ is $\theta_{S / \rho}$-reduced.

THEOREM 3.2. Let $S=S^{0}$ be a completely 0-simple semigroup. Then $\mu$ (where $\mu$ is the maximum idempotent separating congruence on $S$ ) is $\theta$-reduced. Hence the natural map of $\Lambda(S / \mu)$ onto $\Lambda(S) / \theta$ is a complete lattice isomorphism.

Proof. Since the identity congruence on $S$ is $\left[\{1\}, i_{I}, i_{\Lambda}\right]$, where $i_{I}$ and $i_{\Lambda}$ are the identity equivalence relations on $I$ and $\Lambda$, then $\mu=\left[G, i_{I}, i_{\Lambda}\right]$ by Lemma 3.1. Thus the basic group of $S / \mu$ is $\{1\}$. Consequently no two distinct congruences on $S / \mu$ are $\theta_{S / \mu}$-equivalent and so $S / \mu$ is $\theta_{S / \mu}$-reduced. Since $\theta$ is a congruence on $\Lambda(S)$, it follows that $\mu \subseteq \bigvee\{\sigma \in \Lambda(S): \sigma \in \rho \theta\}$ for each $\rho \in \Lambda(S)$. Let $(\rho, \sigma) \in \theta$ with $\rho=\left[N, \tau_{I}, \tau_{\Lambda}\right]$ and $\sigma=\left[M, \tau_{I}, \tau_{\Lambda}\right]$. If, in addition, $\mu \subseteq \rho, \sigma$, then $N=M=G$ and so $\rho=\sigma$. Thus $\rho / \mu \rightarrow \rho \theta$ is a complete lattice isomorphism of $\Lambda(S / \mu)$ onto $\Lambda(S) / \theta$.

THEOREM 3.3. Let $S=S^{0}$ be a primitive regular semigroup. Then $\Lambda(S)$ is semimodular. Further, $\mu$ is $\theta$-reduced and so $\Lambda(S / \mu)$ and $\Lambda(S) / \theta$ are completely lattice isomorphic.

Proof. By [6, Theorem 1], $S$ is a 0-direct union of completely 0-simple subsemigroups $\left\{S_{i}: i \in I\right\}$. That is, $S$ is the 0 -disjoint union of $\left\{S_{i}: i \in I\right\}$ and $S_{i} S_{j}=\{0\}$ if $i \neq j$. From this it follows that $\rho \rightarrow \prod_{i \in I}\left(\rho \mid S_{i}\right)$ is an order preserving bijection of $\Lambda(S)$ onto $\prod_{i \in I} \Lambda\left(S_{i}\right)$. Thus $\Lambda(S)$ is semimodular since semimodularity is preserved by direct products. Further, $(\rho, \sigma) \in \theta_{S}$ if and only if $\left(\rho\left|S_{i}, \sigma\right| S_{i}\right) \in \theta_{S_{i}}$ for each $i \in I$. Hence

$$
\mu=\left\{(x, y) \in S \times S:(x, y) \in \mu_{i} \text { for some } i \in I\right\},
$$

where $\mu_{i}$ is the maximum idempotent separating congruence on $S_{i}$, and so $\mu$ is $\theta$-reduced. 


\section{REFERENCES}

1. A. H. Clifford and G. B. Preston, The algebraic theory of semigroups, Amer. Math. Soc. Math. Surveys No. 7, Vol. 1 (Providence, R.I., 1961).

2. J. M. Howie, The lattice of congruences on a completely 0-simple semigroup (unpublished).

3. G. Lallement, Congruences et équivalences de Green sur un demi-groupe régulier, C. R. Acad. Sci. Paris, Sér. A-B 262 (1966), A613-A616.

4. G. Lallement, Demi-groupes réguliers, Thesis, Paris (1966).

5. G. B. Preston, Chains of congruences on a completely 0-simple semigroup, J. Australian Math. Soc. (1) 6 (1966), 76-82.

6. G. B. Preston, Matrix representations of inverse semigroups; to appear.

7. D. Rees, On semigroups, Proc. Cambridge Philos. Soc. 36 (1940), 387-400.

8. N. R. Reilly and H. E. Scheiblich, Congruences on regular semigroups, Pacific J. Math. 23, (1967), 349-360. 275.

9. T. Tamura, Decompositions of a completely simple semigroup, Osaka Math. J. 12 (1960), 269-

University of South Carolina

COLUMBIA

South Carolina 